This item was submitted to Loughborough's Research Repository by the author.

Items in Figshare are protected by copyright, with all rights reserved, unless otherwise indicated.

\title{
Effect of surface topography upon micro-impact dynamics
}

PLEASE CITE THE PUBLISHED VERSION

http://dx.doi.org/10.1088/2051-672X/4/1/014001

\section{PUBLISHER}

(C) IOP science

\section{VERSION}

AM (Accepted Manuscript)

\section{PUBLISHER STATEMENT}

This work is made available according to the conditions of the Creative Commons Attribution-NonCommercialNoDerivatives 4.0 International (CC BY-NC-ND 4.0) licence. Full details of this licence are available at: https://creativecommons.org/licenses/by-nc-nd/4.0/

\section{LICENCE}

CC BY-NC-ND 4.0

\section{REPOSITORY RECORD}

Mohammadpour, Mahdi, Nick Morris, Michael Leighton, and Homer Rahnejat. 2015. "Effect of Surface Topography Upon Micro-impact Dynamics". Loughborough University. https://hdl.handle.net/2134/18975. 


\title{
Effect of Surface Topography upon Micro-Impact Dynamics
}

\author{
M. Mohammadpour, N.J. Morris, M. Leighton and H. Rahnejat \\ Wolfson School of Mechanical \& Manufacturing Engineering, Loughborough University, \\ Loughborough, UK \\ Corresponding Author: H.Rahnejat@lboro.ac.uk
}

\begin{abstract}
Often the effect of interactions at nano-scale determines the tribological performance of load bearing contacts. This is particularly the case for lightly loaded conjunctions where a plethora of short range kinetic interactions occur. It is also true of larger load bearing conjunctions where boundary interactions become dominant. At the diminutive scale of fairly smooth surface topography the cumulative discrete interactions give rise to the dominance of boundary effects rather than the bulk micro-scale phenomena, based on continuum mechanics. The integration of the manifold localised discrete interactions into a continuum is the pre-requisite to the understanding of characteristic boundary effects, which transcend the physical length scales and affect the key observed system attributes. These are energy efficiency and vibration refinement. This paper strives to present such an approach. It is shown that boundary and near boundary interactions can be adequately described by surface topographical measures, as well the thermodynamic conditions.
\end{abstract}

\section{Keywords}

Adhesion, Capillary Action, micro-Impact dynamics

\section{Nomenclature}
A Apparent contact area
D Gap between an asperity and a flat surface
$E^{*} \quad$ Equivalent modulus of elasticity of two contacting surfaces, $E^{*}=\frac{1}{\left(\frac{1-v_{1}^{2}}{E_{1}}+\frac{1-v_{2}^{2}}{E_{2}}\right)}$ 


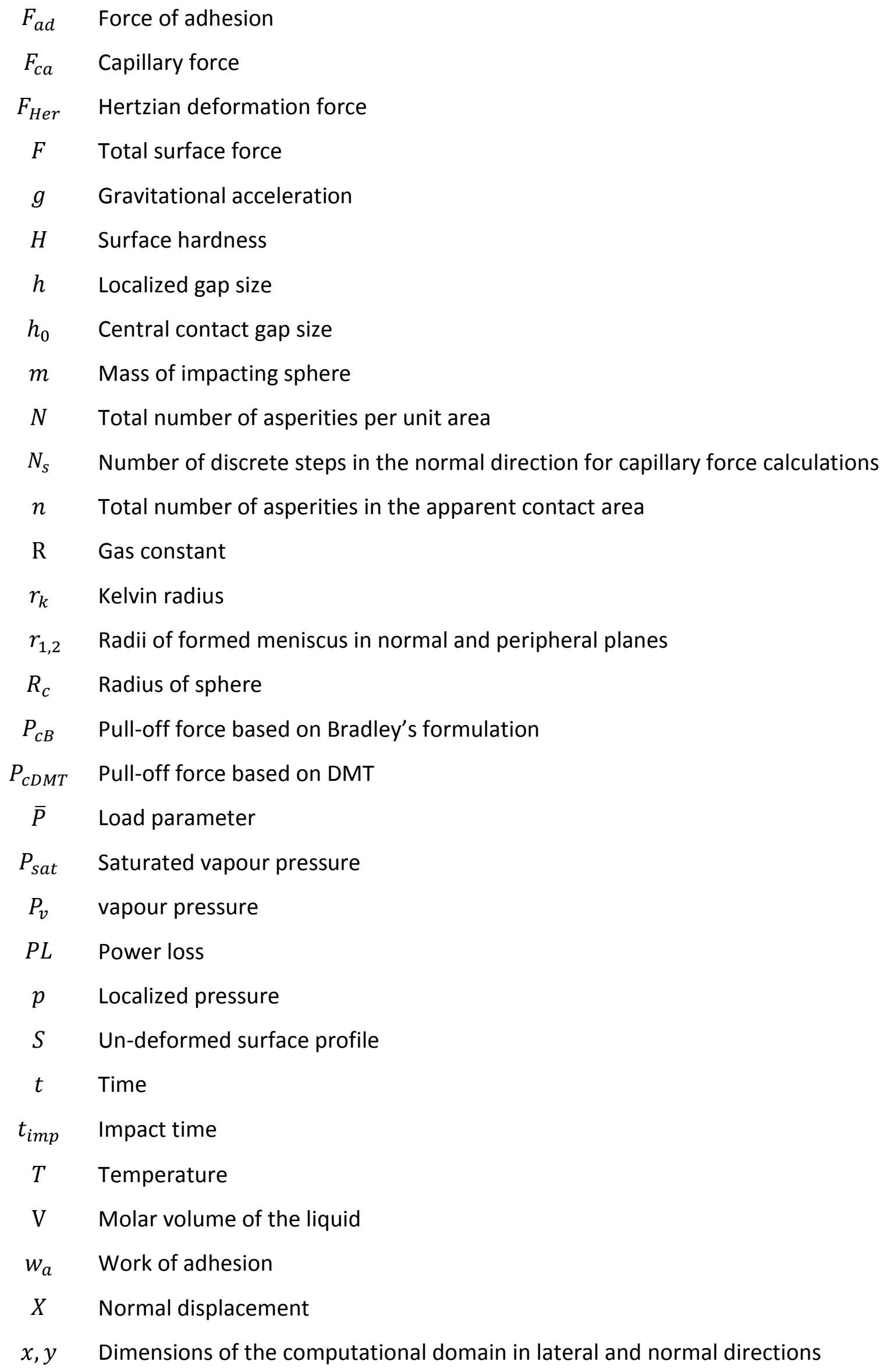

$x, y \quad$ Dimensions of the computational domain in lateral and normal directions 
Greek symbols:

$\beta \quad$ Equivalent summit radius of an opposing asperity pair

$\delta \quad$ Localised deformation

$\delta_{0} \quad$ Deformation at the centre of the contact

$\varepsilon \quad$ The interatomic spacing

$\varepsilon_{p} \quad$ Pressure convergence tolerance

$\gamma_{l v} \quad$ Liquid-vapour interfacial energy

$\mu \quad$ Tabor's number

$\psi \quad$ Plasticity index

$\theta \quad$ Contact angle of water with the surface

$\lambda \quad$ Elasticity parameter

$\lambda^{\prime} \quad$ Stribeck's parameter

$\sigma \quad$ Composite standard deviation of asperity heights' distribution

\section{1- Introduction}

There has been a growing trend in component miniaturisation for all forms of mechanisms and devices. This approach has gathered pace in recent times because of two important reasons. Firstly, there has been a drive towards personalisation of many devices, where compactness for ease of mobility is seen as paramount. Secondly, reduction of energy consumption, whilst enhancing performance is also regarded as a key attribute. In many cases a repercussion of component miniaturisation has been the corresponding diminutive dimensions of load bearing conjunctions, often operating under relatively light loads. As Dowson [1] has observed, the occurrence of nano-scale lubricant films has become commonplace. An important point is that the behaviour of lightly loaded ultra-thin film conjunctions, often operating at very high speeds, deviates from the classical tribological theories developed for micro-scale conjunctions. No longer the prevailing kinetics at the micro-scale, such as viscous and deformation forces dominate the interactions in the scale of minutiae [2]. Remarkably, Feynman predicted that in such diminutive conjunctions the 
effect of viscosity would become so dominant as to make them inoperable, thus advising: Let the bearing run dry [3]. However, adhesion in fairly smooth gaps has become one of the major concerns. This means that nano-scale ultra-smooth surfaces must necessarily operate at very high speeds, as also envisaged by Feynman [3]. This is already true of all microelectromechanical systems (MEMS), even for relatively rough surfaces of fairly small contacts $[4,5]$.

Adhesion also plays the same role at the scale of asperity pair summit interactions in the more commonly encountered micro-scale contacts. However, in most cases a form of liquid intervenes or is entrapped between the topography of the counter faces, thus reducing the surface energy of adhesion. This may be a lubricant in many cases or simply water condensation, forming a mono-layer on any exposed surface in a short period of $25 \mu \mathrm{s}$, growing exponentially then after [6]. At the same time the presence of lubricant in contact of rough surfaces can lead to capillary adhesion through the formation of micro-menisci $[7,8]$. Other kinetic laws can also be present such as hydration in conjunctions of hydrophobic nature or solvation in lightly loaded molecularly smooth contacts [9-11], or indeed long range van der Waal's interactions and electrostatic repulsion $[9,12]$. Therefore, unlike the well-understood and observed micro-scale contacts such as hydrodynamic/elastohydrodynamic phenomena [12, 13], the interactions at the lower scales and underlying to the small regions of larger micro-scale contacts herald a plethora of less understood phenomena. This lack of understanding is of concern as it ultimately affects important issues such as load carrying capacity, friction, wear and deformation, all with important practical implications.

This paper studies the effect of deformation and adhesion at the small scale of fairly smooth wetted surface topography which is underlying to the operational integrity of many machines and devices whether in micro-mechanisms or as a part of a macro-scale system. In particular, small scale impact of surface features in wet environment is investigated.

Although the concept of using statistical distribution of surface characteristics has been used in earlier works; but these works rarely include the effect of adhesion and capillary effects which are significantly effective in the scale of minutiae. This paper takes in to account the effect of all effective forces using the same statistical approach. 
This is often of interest when the adhesion can potentially play a positive role as an energy sink, reducing vibrations [8]. Conversely, it can also promote stiction and loss of functionality in miniaturised devices such as in MEMS [4]. Therefore, an optimum balance of kinetic interactions is sought, which clearly calls for a better fundamental understanding.

The paper also presents modified and pre-estimated integrals of statistical distributions. This extensively reduces the computational costs of the simulation without sacrificing the accuracy. Therefore the tribological method can be integrated in the dynamic model. This reasonably fast and inclusive integrated approach enables the whole model to predict the above mentioned conundrum between the tribological performance and the dynamic refinement. This is a subject which is highlighted in this paper and has not been reported hitherto.

\section{2- Nano-scale Impact Dynamics}

The case of impact dynamics of a silica $\left(\mathrm{SiO}_{2}\right)$ micro-sphere of radius $50 \mu \mathrm{m}$ against a silicon carbide ( $\mathrm{SiC}$ ) surface; a standard sample for calibration of atomic force microscopes, is investigated. Figure $1(\mathrm{a})$ and (b) show images of the $\mathrm{SiC}$ sample and the $\mathrm{SiO}_{2} \mathrm{micro}^{-s p h e r e}$ obtained by a Leica DM6000M microscope.

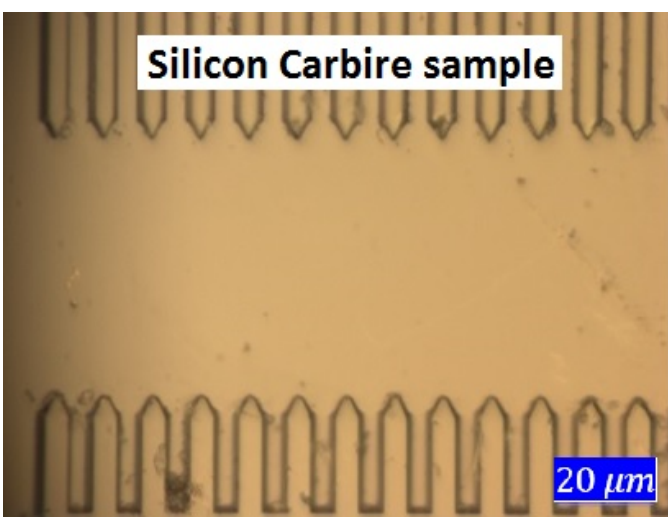

(a)

\section{Silica Micro Sphere}

$20 \mu m$

(b

Figure 1: The components of the investigated impact dynamic investigation

The surface topography is measured using an infinite focus white light interferometer with measurement repeatability of $\pm 1 \mathrm{~nm}$. The peak height distribution is calculated using the convolution of the two surface topographies in contact at varying separations (figure 2). 
The measured asperity height distribution is shown in figure 3 . This is compared with a standard Gaussian distribution. It is evident that the distribution of composite real surface topography conforms quite well to a Gaussian distribution. Therefore, the use of analysis method expounded in section 3 is justified.

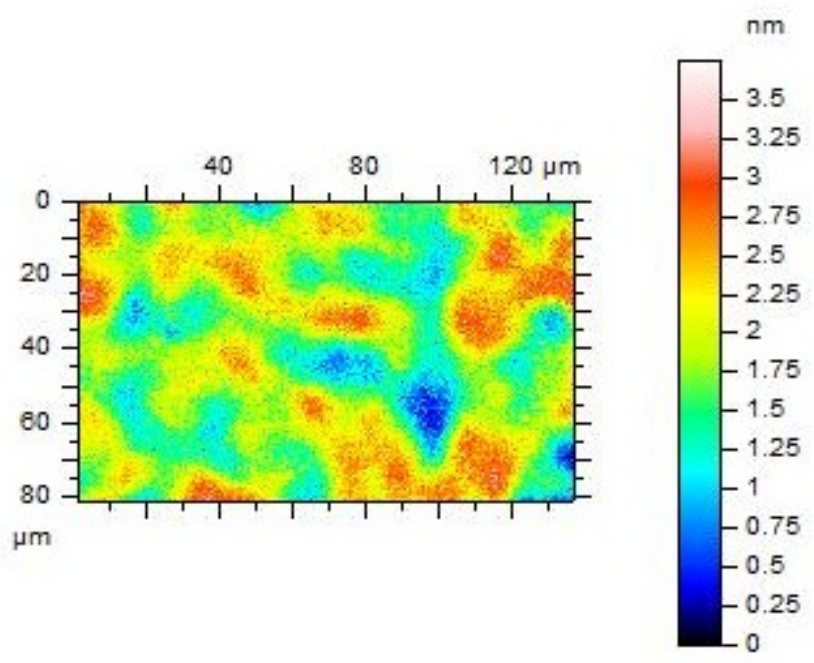

Figure 2: Post-processed surface height distribution

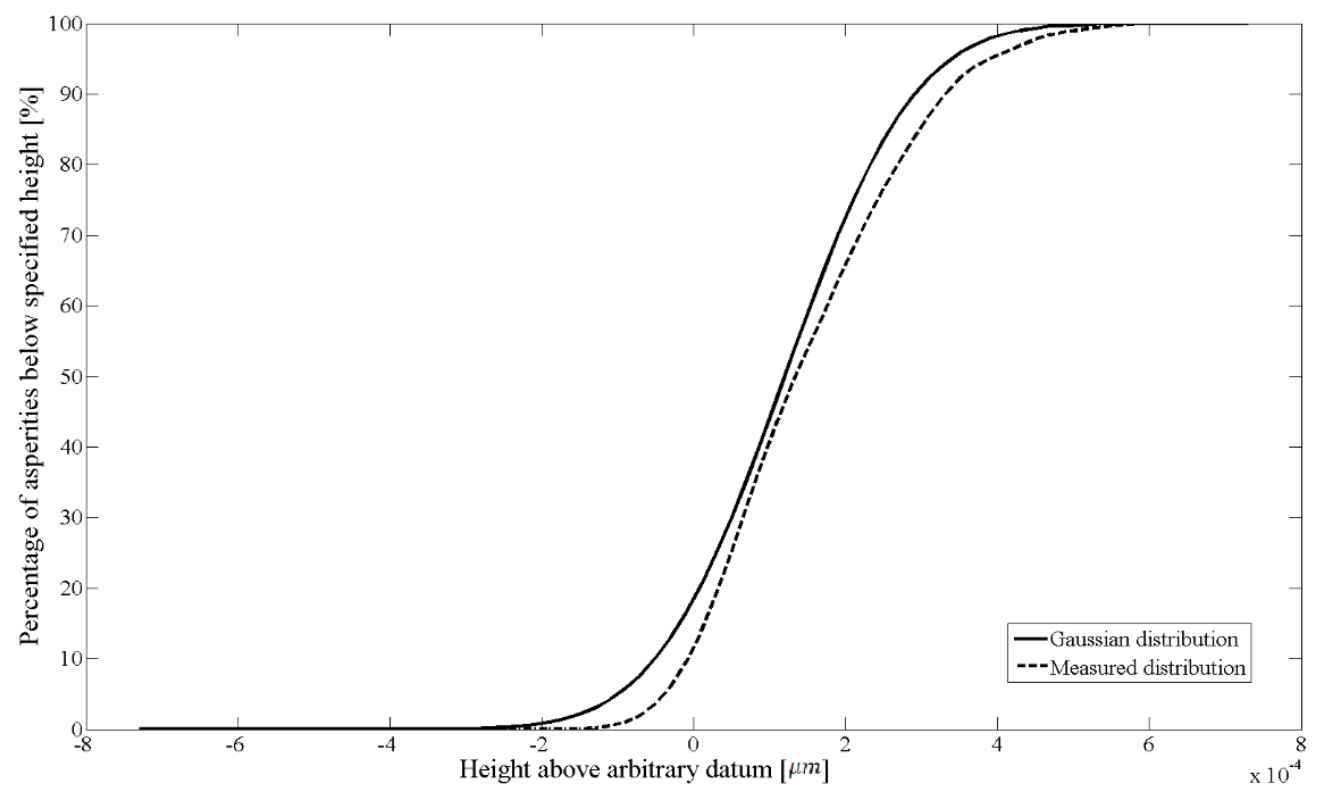

Figure 3: Conformity of measured convoluted surface height distribution to Gaussian

\section{3- Method of Analysis}




\section{1- Background Theory}

The continuum mechanics of elastic and plastic contact of a dry single asperity is now fairly well established. Bradley [14] showed that the required force to separate two rigid spheres can be expressed as:

$P_{c B}=2 \pi \beta w_{a}$

where $\beta$ is the equivalent radius of an asperity pair in normal contact and $w_{a}$ is the work of adhesion. Later, Johnson et al [15] and Derjaguin et al [16] presented models for adhesion force of deformable spheres (these are referred to as the JKR and DMT models respectively). These models provide different estimations of adhesion force. Later, Tabor [17] suggested that both models are appropriate for different levels of deformation (i.e. asperity pair stiffness). He presented the Tabor number as:

$\mu=\left(\frac{\beta w_{a}^{2}}{E^{2} \varepsilon^{3}}\right)^{1 / 3}$

where $E^{*}$ is the equivalent elastic modulus of the contacting asperities and $\varepsilon$ is the interatomic spacing. The Tabor number can be interpreted as the ratio of the elastic deformation to the range of action of the adhesive force. Therefore, the larger the Tabor number, the greater the extent of deformation, or a more compliant asperity pair contact. If the Tabor number exceeds 5 , the JKR model is a better representation, whilst for a value less than 0.1 , the use of DMT model is more appropriate. The intermediate region was explored, initially by Muller et al. [18] and later in more detail by Greenwood [19]. A generic model was finally developed by Maugis-Dugdale [20] (referred to as the M-D model). Since all these models assume elastic deformation of asperities, in the presence of any inelastic-viscoelastic or plastic deformation, alternative approaches need to be employed [21, 22]. Greenwood and Williamson [23] derived a dimensionless group, referred to as plasticity index for ascertaining the state of deformation of rough surfaces. For the case of assumed spherical asperities of exponential surfaces:

$\psi=\frac{E^{*}}{H} \sqrt{\frac{\sigma}{\beta}}$ 
where, $H$ is the indentation hardness and $\sigma$ is the standard deviation of the asperity heights' distribution. A value of index exceeding unity points to some yielding of asperities. Therefore, use of a simple index together with the Tabor's parameter can form the basis for the choice of an adhesion model for any analysis. However, the index is independent of load, and is based on the yield strength of the softer of the two counter face material.

\section{2- Dry Adhesion}

Alternatively, the adhesion map (figure 4) can be used, which takes into account the effect of applied load (in this case the impact force). The vertical axis on this map is the ratio of the applied load to the adhesive force, and is referred to as the load parameter, $\bar{P}$ [24]. For all the dry elastic adhesive models the value of this ratio is less than 100 , otherwise adhesion is deemed to be negligible in comparison with the deformation force (Hertzian for the assumed asperities as ellipsoidal elastic solids). For a single asperity the suitable dry adhesion model can be defined by the load ratio and the elasticity parameter: $\lambda=1.16 \mu$ [24]. Therefore, for any given applied load and measured asperity geometry and mechanical properties, the most appropriate adhesion model can be adopted.

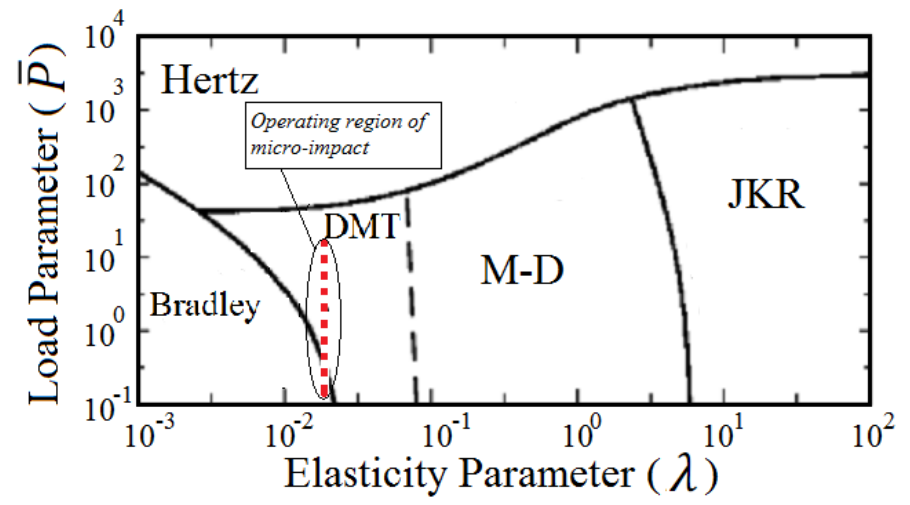

Figure 4: The adhesion map [24] with typical operating region of the current analysis results

Figure 4 shows the typical operating region of the impact of the $\mathrm{SiO}_{2}$ micro-sphere with the SiC sample in all the analysis carried out in section 6 . 
All the noted adhesion models are based on continuum mechanics. Binquan and Robbins [25] presented an atomic simulation of the single asperity contact and compared it with the continuum mechanics models, showing that the behaviour in bulk could adequately be described through continuum mechanics down to the scale of 2-3 atomic diameters.

There is also the deformation of asperities to be considered, usually using the classical Hertzian theory. This aspect is studied in some detail by Greenwood and Trip [26]. They showed that the assumption of an equivalent asperity against a flat surface provides good accuracy despite its relative simplicity. Finally, they used a Gaussian distribution of asperity heights in order to expand the single asperity model to an entire rough surface. This is the approach also used in the current analysis.

In the current analysis, the data used is provided in Table 1. For the combined topography and mechanical properties of the micro-sphere and the SiC flat sample, the calculated Tabor number is: 0.02 , which is less than 0.1 , with the elasticity parameter: $\lambda \approx 0.0235$, being independent of the applied load. This is clear from figure 4. Therefore, the DMT model is used for the current analysis.

Table 1: Relevant Mechanical, topographical and interfacial properties

\begin{tabular}{|l|l|}
\hline Young's modulus of $\mathrm{SiO}_{2}$ & $80 \mathrm{GPa}$ \\
\hline Young modulus of $\mathrm{SiC}$ & $161 \mathrm{GPa}$ \\
\hline Poisson's ratio of $\mathrm{SiO}_{2}$ & 0.25 \\
\hline Poisson's ratio of $\mathrm{SiC}$ & 0.23 \\
\hline Number of asperities per unit area & $169 \times 10^{14} \mathrm{~m}^{-2}$ \\
\hline Work of adhesion, $w_{a}$ & $30 \mathrm{~mJ} / \mathrm{m}^{2}[27]$ \\
\hline Water vapour interfacial energy, $\gamma_{l v}$ & $75 \mathrm{~mJ} / \mathrm{m}^{2}$ \\
\hline Contact angle for water with the Si surfaces, $\theta$ & $73^{\circ}$ \\
\hline
\end{tabular}

In the DMT adhesion model, the pull-off force required to overcome adhesion of two assumed hemispherical asperities is:

$P_{C D M T}=2 \pi \beta w_{a}$ 
In order to expand this asperity pair interaction into that of the contact of overall rough surfaces, comprising many asperity pairs, a statistical distribution of asperity heights need to be assumed at any separation $d$ (this is the distance between a smooth plane and the equivalent reference plane at the mean height of the asperities). In this study a Gaussian distribution is assumed as the convolution of asperity heights of the two rough surfaces (mico-sphere and the SiC sample) which has already been shown to closely follow a Gaussian distribution (figure 3). If the number of asperities per unit area is $N$ (see Table 1 ), then the total number of asperities per unit area which come into contact at any separation $d$ is:

$n=N \int_{d}^{\infty} \varphi(z) d z$

where, $\varphi(z)$ represents the Gaussian distribution of the heights of the asperities, $z$, which remain above the mean surface height. This is defined as:

$\varphi(z)=\frac{1}{\sigma \sqrt{2 \pi}} \exp \left(-\frac{z^{2}}{2 \sigma^{2}}\right)$

$\varphi(z) d z$ is the probability of height of an asperity residing between $z$ and $z+d z$. To simplify the numerical procedure, one can consider:

$F_{0}\left(\lambda^{\prime}\right)=\frac{1}{\sqrt{2 \pi}} \int_{\lambda^{\prime}}^{\infty} \exp \left(-\frac{\lambda^{\prime 2}}{2}\right) d \lambda^{\prime}$, Stribeck parameter: $\lambda^{\prime}=\frac{z}{\sigma}$

Therefore, from equations (5) - (7) it follows that:

$n=N F_{0}\left(\lambda^{\prime}\right)$

If the apparent contact area is $A$, from equations (4) and (8) the total adhesion force becomes:

$F_{a d}(x, y)=2 \pi A \beta w_{a} N F_{0}\left(\lambda^{\prime}\right)$

$F_{0}\left(\lambda^{\prime}\right)$ can be integrated numerically and a polynomial equation may be obtained through regression of the results as:

$$
\begin{aligned}
& F_{0}\left(\lambda^{\prime}\right)=-0.00004965 \lambda^{\prime 7}+0.00003763 \lambda^{\prime 6}+0.006278 \lambda^{\prime 5}-0.048 \lambda^{\prime 4}+0.1275 \lambda^{\prime 3}- \\
& 0.03592 \lambda^{\prime 2}-0.3912 \lambda^{\prime}+0.5
\end{aligned}
$$


Therefore, the net adhesion of the conjunction can be obtained at any instant during the impact and rebound of the micro-sphere upon the SiC sample, using equations (9) and (10).

\section{3- Capillary Adhesion (Meniscus Action)}

Another source of adhesion is through capillary action. The atmospheric moisture condenses and forms menisci around pairs of surface asperities [6]. Capillary adhesion is usually much larger than other forms of adhesion and should ideally be avoided in order to reduce the chance of stiction. Although this is usually achieved in the case of MEMS devices, the same is not true of micro-scale load bearing conjunctions. Teodorescu and Rahnejat [2, 8] modelled the capillary and dry adhesion, as well as long range van der Waal's interactions together with Newtonian mechanics and classical Hertzian theory. However, they neglected the effect of gap size on the magnitude of the capillary force. Hariri et al [28] presented a method to take into account the capillary adhesion in MEMS' contacts, assuming the contact of a flat rigid surface with an equivalent elastic rough surface.

Figure 5 is a schematic representation of a meniscus bridge formed between an equivalent asperity and a flat surface. It also shows a rough surface, comprising a series of equivalent asperities of a pair of rough surfaces at different summit heights with varying separations from a flat smooth counterface. Thermodynamic equilibrium is assumed between the formed menisci and the environment based on Lord Kelvin's equation [29] as:

$r_{k}=\left(\frac{1}{r_{1}}+\frac{1}{r_{2}}\right)^{-1}=\frac{\gamma_{l v} V}{R T \ln \left(\frac{P_{v}}{P_{s a t}}\right)}$

where, $r_{k}$ is the Kelvin radius, $P_{v}$ the vapour pressure and $P_{s a t}$ the saturated vapour pressure. $T$ is the absolute temperature, $R$ is the gas constant and $V$ is the molar volume of the liquid (in this case water). 


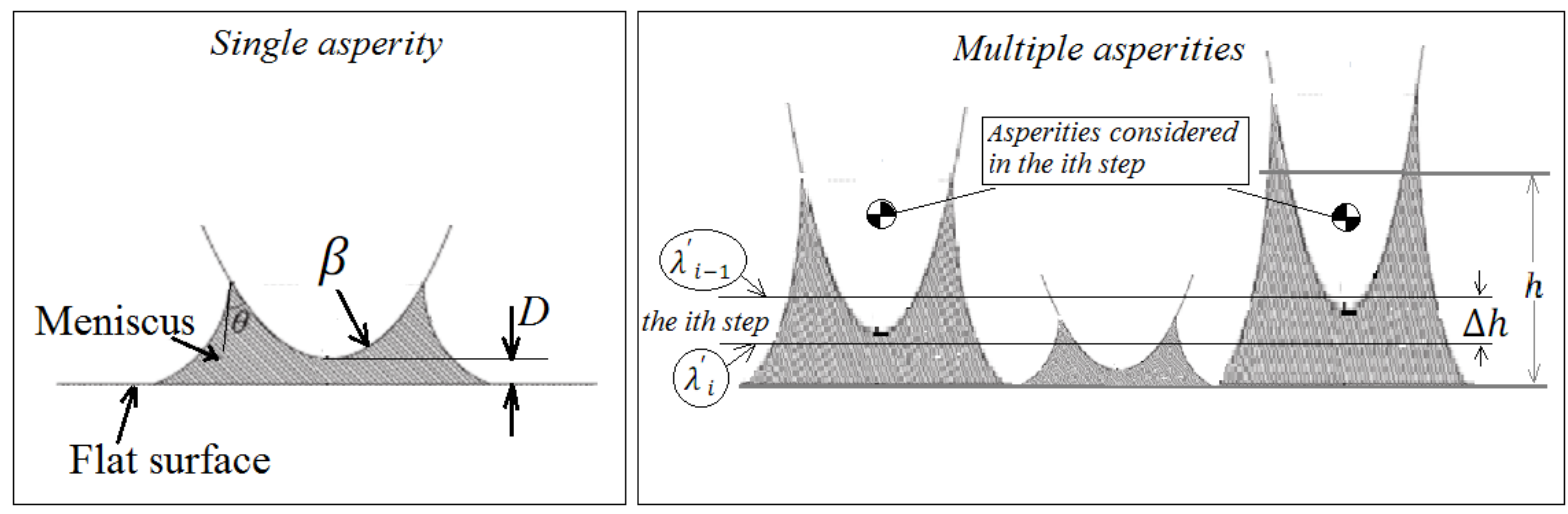

Figure 5: Formation of menisci for a single asperity and an equivalent rough surface

Hariri et al. [28] assumed a parabolic asperity profile and after some manipulation derived the capillary force as:

$f_{c a}=\frac{4 \pi \beta \gamma_{l v} \cos \theta}{1+D /\left(2 r_{k} \cos \theta-D\right)}$

For a Gaussian distribution of asperities, a similar simple equation can be obtained for the capillary force. Equation (12) depends on the gap size, $h$ (as shown in equation (15)). Therefore, for a distribution of asperities gradually entering into contact, the overall separation should be subdivided into a number of incremental steps (figure 5). The step size is:

$\Delta h=h / N_{S}$

Now the number of asperities between successive steps for the unit area of contact can be calculated as:

$n=\frac{N}{\sqrt{2 \pi} \sigma}\left[F_{0}\left(\lambda_{i}^{\prime}\right)-F_{0}\left(\lambda_{i-1}^{\prime}\right)\right]$, where $\lambda_{i}^{\prime}=(h-i \Delta h) / \sigma$

All the asperities encountered between any two successive incremental steps are assumed to have the same gap size. Therefore, the total capillary force for the apparent contact area becomes:

$F_{c a}(x, y)=\sum_{i=1}^{n} \frac{4 \pi A n \beta \gamma_{l v} \cos \theta}{1+i \Delta h /\left(2 r_{K} \cos \theta-i \Delta h\right)}$

\section{4- Asperity Deformation}


Greenwood and Tripp [26] presented an equation in order to calculate asperity deformation using the classical Hertzian theory. They demonstrated the validity of an equivalent rough surface comprising hemispherical asperity profiles against a flat surface. They also showed the same for cylindrical and conical asperities. Using numerical integration approach, they showed that:

$F_{H e r}(x, y)=\frac{4}{3} N \beta^{1 / 2} \sigma^{3 / 2} E^{*} F_{3 / 2}\left(\lambda^{\prime}\right)$

Where $E^{*}$ is the equivalent modulus of elasticity of two contacting surfaces. Using this combined material property, the substrate is assumed as rigid surface and the sphere is considered with this equivalent parameter. Similar approach has been used extensively in the literature [26].

$F_{3 / 2}\left(\lambda^{\prime}\right)$ is the numerically integrated value derived from a Gaussian distribution. A polynomial fit of the numerical results is presented here:

$$
\begin{aligned}
& F_{3 / 2}\left(\lambda^{\prime}\right)=0.0001217 \lambda^{\prime 7}-0.001913 \lambda^{\prime 6}+0.01106 \lambda^{\prime 5}-0.02193 \lambda^{\prime 4}-0.04654 \lambda^{\prime 3}+ \\
& 0.3215 \lambda^{\prime 2}-0.6167 \lambda^{\prime}+0.43
\end{aligned}
$$

One should note that the presented asperity interaction models are based on the assumption of no coalescence of asperities. In some references $[30,31]$ it is demonstrated that this assumption might not be appropriate in all cases. In current paper, the system operates under relatively light loads. In this limit, coalescing of asperities could be neglected. However, interaction between adjacent asperities might affect results in terms of real contact area and total load.

A parabolic contact profile for the micro-sphere is assumed in the contact region. The value of functions $F_{3 / 2}$ and $F_{0}$ virtually diminish for $\lambda^{\prime}=3$. Based on this a sufficiently large computational domain is selected. The profile of the micro-sphere in figure $1(b)$ is expressed as:

$S(x, y)=x^{2} / 2 R_{C}$

where $R_{c}=50 \mu \mathrm{m}$ is the radius of the sphere and $x, y$ are the dimensions of the computational domain. $S$ is the un-deformed parabolic contacting profile. Under the net 
adhesive and deformation forces at each point within the micro-scale computational domain, the localised deformation is computed as [12]:

$\delta(x, y)=\frac{1}{\pi E^{*}} \sum \sum \bar{D}(i, j) p(k, l)$

where: $p(k, l)=F(k, l) / A(k, l)$.

$A(k, l)$ is the computational grid area which is the apparent contact area in equations (9). The forces $F(k, l)$ are calculated for each grid point and summed over the entire computational domain for the impact dynamics' analysis. The instantaneous gap size at each localised position $x, y$ is obtained as:

$h(x, y)=h_{0}+S(x, y)+\delta(x, y)-\delta_{0}$

This value is used in equations (9), (15) and (16) in $\lambda^{\prime} . h_{0}$ is the separation at the centre of the impact zone (at the minimum separation) and $\delta_{0}$ is the corresponding deformation there (figure 6).
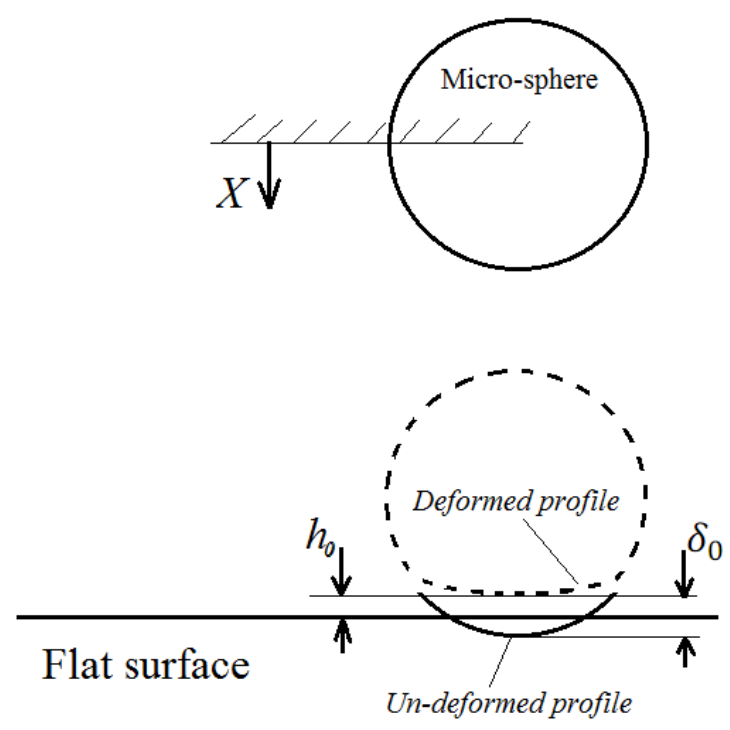

Figure 6: Geometry of the conjunction

\section{5- Micro-impact Dynamics}

The impact problem is considered as a one-degree-of-freedom system in this case. This means that any slight rotation of the micro-sphere during the impact and rebound is 
ignored. Any material hysteretic damping through the deformation of asperities is also neglected. In practice, there can be some generated friction, causing the rotation of the sphere. The forces acting upon the sphere are the cumulative effect of adhesion, capillary action and Hertzian deformation forces at the diminutive level of the asperities, as well as the body force due to gravity. The equation of motion becomes:

$m(\ddot{X}-g)=\sum F$

where: $\sum F=\sum \sum F_{a d}(x, y)+\sum \sum F_{c a}(x, y)-\sum \sum F_{H e r}(x, y)$

where, $m$ is the mass of the micro-sphere and $g$ is the gravitational acceleration.

\section{4- Method of Solution}

The equation of motion is solved by a $4^{\text {th }}$ order Runge-Kutta integration algorithm. The integration error is set to $10^{-10}$. The time step is chosen as $0.1 \mu \mathrm{s}$ due to the very fast nature of the micro-impact problem. Therefore the time history of the impact event can be captured properly. At each time step, the following calculation steps are undertaken:

1. An initial gap shape is calculated for each grid point of the domain using equation (20) for an un-deformed geometry.

2. The forces acting at each grid point are calculated using equations (9), (15) and (16).

3. The generated pressure and deformation are calculated using equation (19).

4. The gap shape in step 1 is now updated using the newly calculated deformation and all the subsequent steps are repeated. This process is continued until the desired error tolerance for the generated pressure distribution is satisfied:

$$
\sum_{A} \frac{\left|p_{\text {new }}-p_{\text {old }}\right|}{p_{\text {old }}} \leq \varepsilon_{p}
$$

where, typically: $10^{-1} \leq \varepsilon_{p} \leq 10^{-3}$.

5. After pressure convergence, the net force $\sum F=\sum \sum p(k, l) A(k, l)$ is calculated by integrating the pressure distribution over the computational domain. This force is used in the equation of motion (21). 
In this manner the effect of nano-scale interactions through to micro-scale impact dynamics is ascertained.

\section{5- Results and Discussion}

Simulations are performed for the $\mathrm{SiO}_{2}$ micro-sphere impacting the $\mathrm{SiC}$ sample plate (section 2). In the simulation process, the impact of an equivalent sphere with equivalent composite surface roughness against a flat smooth and rigid surface is studied. The necessary data for the analysis is provided in table 1.

The presented case study is a representative case of practical applications. An example of such application is the micro-gear conjunction presented in [4]. Due to generality of the presented approach in this paper, it can be used in such applications knowing the geometry and topography of conjunctions. Based on the topography of the surface, equations (9), (15) and (16) should be amended for each case study. Equation (18) is also defined based on the geometry of the conjunction. The material property and the surface topography also specify the right adhesion model to be used from the adhesion map in the figure (4).

In this paper two objectives are pursued in any dynamic system. These are vibration refinement and reduction of power loss; attributes which are often contradictory in nature. In micro- systems, the power loss is due to adhesion and capillary action under the assumed environmental conditions (thermodynamics). For a displacement $X$ of the micro-sphere, it follows that:

$P L=\frac{\int_{0}^{X_{0}}\left[\sum F_{a d}(x, y, X)+\sum F_{c a}(x, y, X)\right] d X}{t_{i m p}}$

where, $t_{i m p}$ is the impact time.

In the case of impact conditions, a larger power loss constitutes higher damping, thus an improved level of vibration refinement. A higher degree of power loss also indicates a greater risk of stiction, thus requiring a larger energy input to sustain the system operation. This is a commonly encountered problem with small scale devices, such as in MEMS [4, 32], where they are required to operate at high speeds in order to reduce the chance of adhesion. 
Figure 7 shows the significant effect of capillary adhesion upon power loss in the scale of minutiae. There is quicker reduction in the amplitude of successive micro-sphere rebounds with the inclusion of moisture than under an assumed dry impact. The comparison is an indication of capillary adhesion's contribution to damping, thus improving vibration refinement. A good indication of this trend is the logarithmic decrement, being the natural logarithm of the ratio of successive micro-sphere rebound amplitudes:

$L D=\ln \frac{X(t)}{X\left(t+t_{i m p}\right)}$

The results in figure 7a show that the logarithmic decrement is 200 times larger in the case of a wet impact at $100 \%$ relative humidity than under dry impact condition. This shows the dramatic damping effect of capillary adhesion. Clearly, it also demonstrates a greater chance of stiction. This is a good example of noted stiction problems with any ingression of moisture into MEMS devices.

Figure $7 \mathrm{~b}$ shows the time history of different acting forces in the system. It shows the fast event of the impact which is captured by the simulation in case of figure $7 a$.

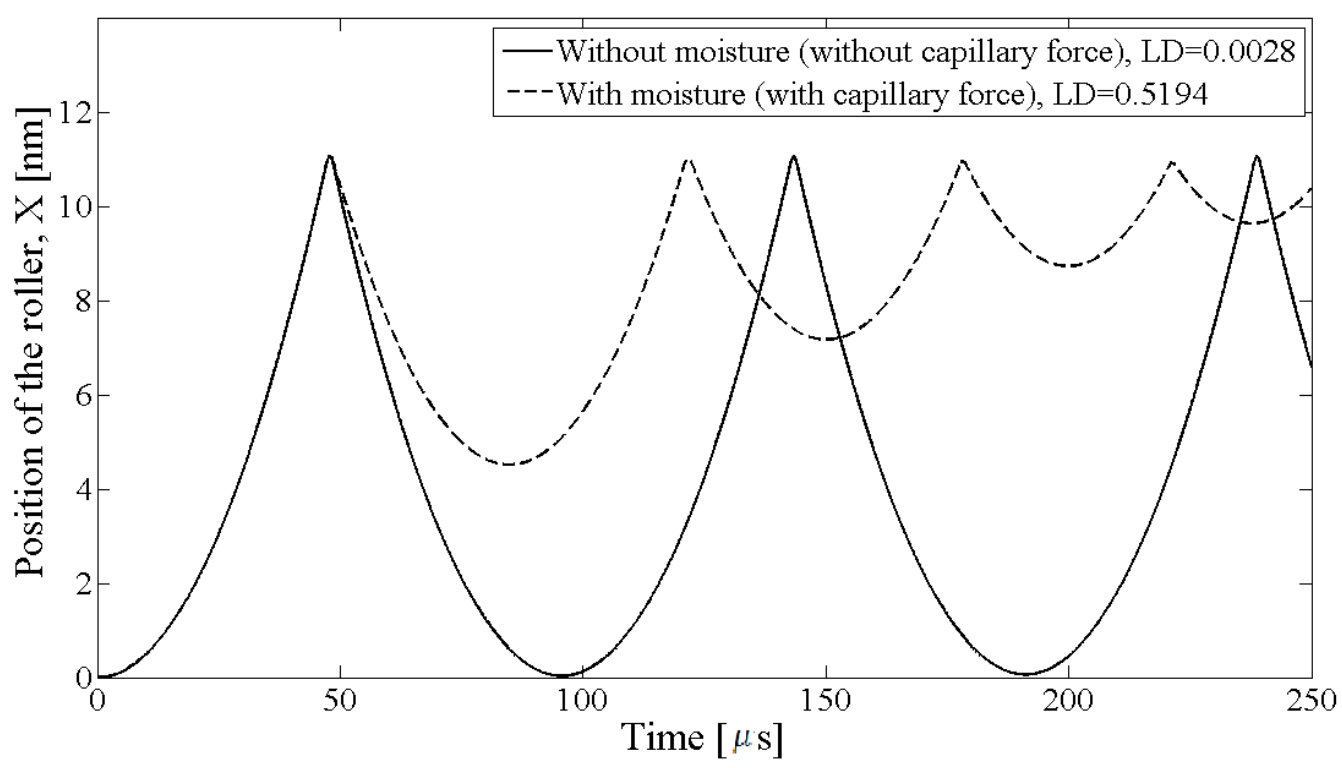

Figure 7a: Effect of the existence of moisture on the impact dynamics 


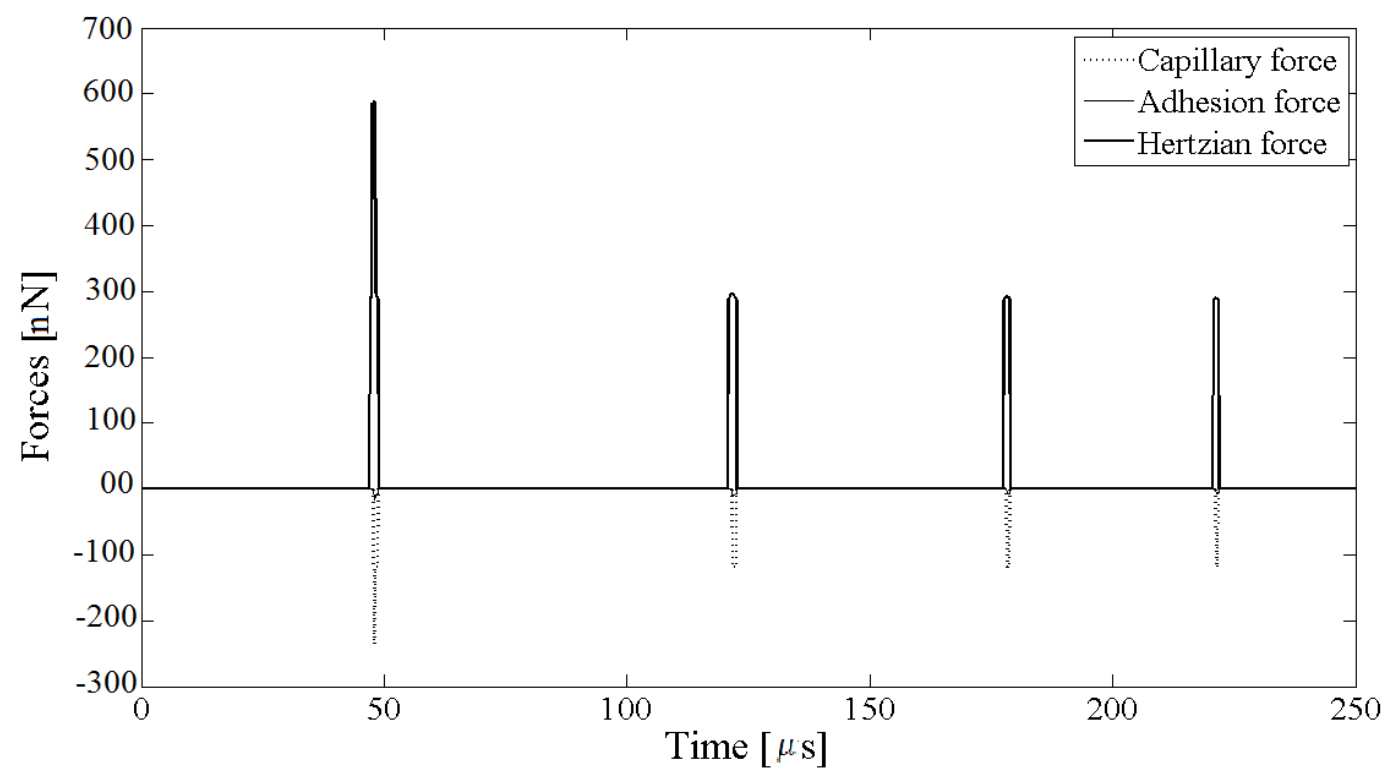

Figure 7b: Time history of acting forces for the case of figure 7a

The ratio $\frac{\beta}{\sigma}$ is a measure of asperity slope (shape). Larger values of this ratio correspond to broader and flatter rough topography. Various surfaces can almost be classified in this manner, depending on material type and method of manufacture/fabrication. Different surface treatments such as coatings, etching, cross-hatching and honing, to name but a few, are used in order to engineer surface topography to suit particular applications which are mainly subject to a boundary or mixed regime of lubrication $[33,34]$. In order to study the effect of these surface parameters upon the impact behaviour, the influence of average asperity summit radius; $\beta$ is shown in figure 8 . Three values of $\beta=0.2,0.25$ and 0.3 are used, all with the same value of $\sigma$. The impact time histories in figure 8 show that for an increasing value of $\beta$ (thus broader and flatter asperities) there is greater loss of impact energy (i.e. a larger value of logarithmic decrement, Table 2). With shallower asperities (i.e. relatively smoother surfaces, constituting a larger asperity summit area) there is an increasing chance of adhesion. This is an expected outcome. In addition to $L D$, the stiction period is also provided in table 2 . The stiction period is the time which the sphere takes to completely attach to the surface. It is predicted by zero velocity as well as zero acceleration. Similar to $L D$, this is a measure of the damping and also the chance of stiction.

The converse is true with an increasing value of $\sigma$, whilst keeping a constant value of $\beta$, rendering sharper asperity features (figure 9). In this case the impact occurs quicker with 
smaller displacements (rebounds) of the micro-sphere. Therefore, smaller amplitude rebounds occur, although the rate of displacement decay is quicker with the smaller values of $\sigma$, or larger ratios: $\frac{\beta}{\sigma}$ as in the previous case. This is indicated by the higher values of logarithmic decrement; $L D$ (Table 3). Therefore, vibration attenuation, a function of $L D$, is directly related to the surface topography, represented by the ratio $\frac{\beta}{\sigma}$, which alters the dry and capillary-induced adhesion contributions.

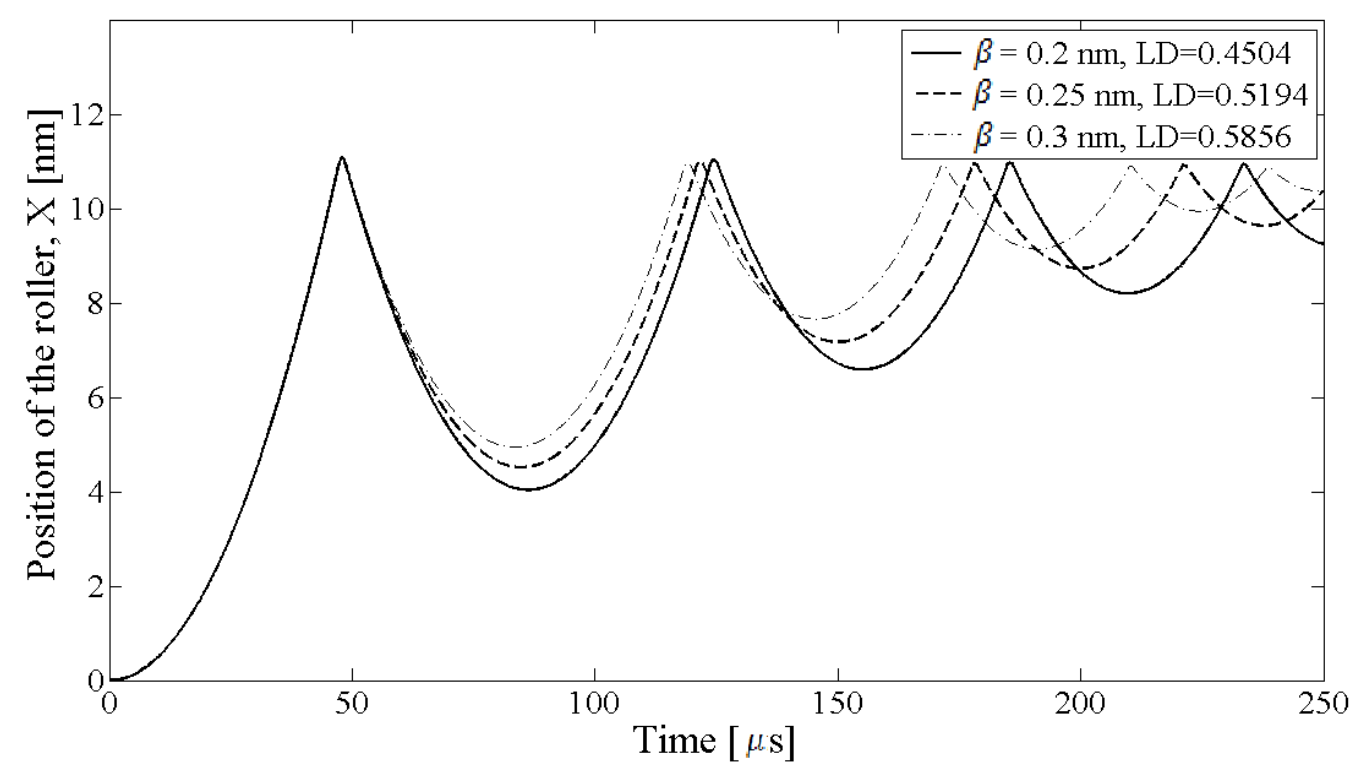

Figure 8: The effect of asperity summit radius, $\beta$ on impact dynamics

Adhesion in both its forms leads to the loss of impact energy and may be interpreted as friction as an energy sink. This means that a reduction in the value of $L D$, whilst attenuating vibration gives rise to frictional losses. Therefore, for all systems, adhesion at all physical scales leads to a degree of energy inefficiency. As already noted no slip condition is assumed at the interface between the micro-sphere and the flat plate at the instant of impact in the current analysis. Also, the equivalent composite asperities are assumed to be subjected to adhesion and deformation normal to the surface of the smooth counter face. In practice, some of the asperities on any pair of rough surfaces interact in an oblique manner, which with any relative sliding motion are subject to ploughing of the softer material. This phenomenon is known as deformation friction which further contributes to the loss of energy [12]. 
Table 2: Stiction period, power loss and $L D$ for different values of $\beta$

\begin{tabular}{|l|l|l|l|}
\hline$\beta$ & $\begin{array}{l}\text { Stiction period } \\
{[\mu \mathrm{s}]}\end{array}$ & $\begin{array}{l}\text { Power loss [ } \\
\left.10^{-4} \mathrm{nW}\right]\end{array}$ & Logarithmic decrement \\
\hline$\beta=0.2 \mathrm{~nm}$ & 392 & 2.0201 & 0.4504 \\
\hline$\beta=0.25 \mathrm{~nm}$ & 337 & 2.0554 & 0.5194 \\
\hline$\beta=0.3 \mathrm{~nm}$ & 300 & 2.2787 & 0.5856 \\
\hline
\end{tabular}

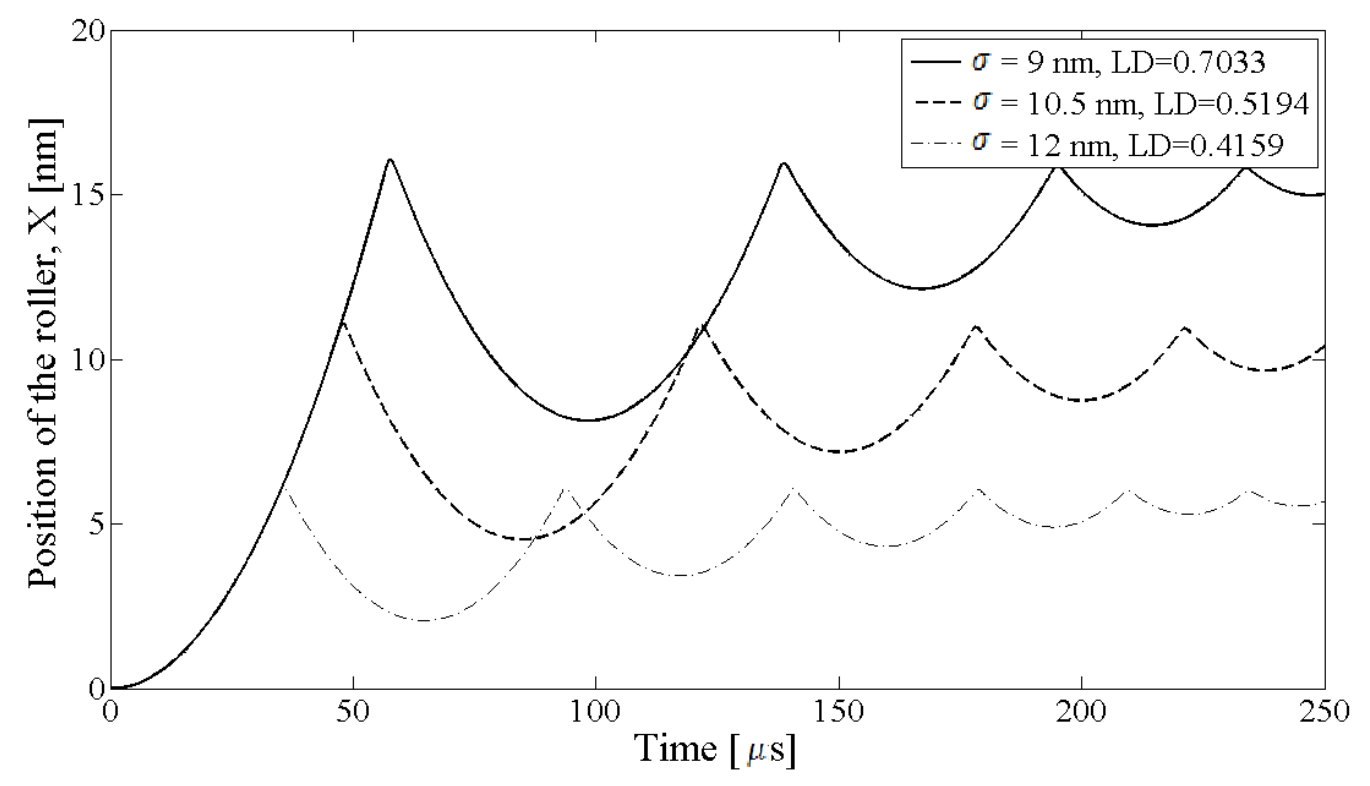

Figure 9: The effect of surface roughness, $\sigma$ on impact dynamics

Table 3: Stiction period, power loss and $L / D$ for different values of $\sigma$

\begin{tabular}{|l|l|l|l|}
\hline$\sigma$ & $\begin{array}{l}\text { Stiction period } \\
{[\mu s]}\end{array}$ & $\begin{array}{l}\text { Power loss [× } \\
\left.10^{-8} \mathrm{nW}\right]\end{array}$ & Logarithmic decrement \\
\hline$\sigma=9 \mathrm{~nm}$ & 307 & 3.2994 & 0.7033 \\
\hline
\end{tabular}




\begin{tabular}{|l|l|l|l|}
\hline$\sigma=10.5 \mathrm{~nm}$ & 337 & 2.0554 & 0.5194 \\
\hline$\sigma=12 \mathrm{~nm}$ & 321 & 1.1648 & 0.4159 \\
\hline
\end{tabular}

\section{6- Conclusions}

The paper investigates the effect of nano-scale interactions through to micro-scale impact dynamics. The impact of a molecularly smooth $\mathrm{SiO}_{2}$ micro-sphere upon an $\mathrm{SiC}$ sample is studied. The analysis includes the effect of localised deformation of micro-sphere of equivalent composite topography and mechanical properties, as well the cumulative effect of asperity adhesion and capillary action under normal atmospheric conditions. Tabor, elasticity and load parameters as well as plasticity index, are used to select the correct adhesion model (in this case DMT) to accurately represent dry adhesion characteristic for the studied case, using the adhesion map. Additionally, simple thermodynamic balance and convolution of measured surface topography of the impacting surfaces are used to develop an overall capillary adhesion model. These sources of adhesion as well as localised deformation of rough topography are used in the impact dynamics analysis. It is shown that for the lightly loaded contact/impact of surfaces of given material combination, surface topographical measures determine the characteristic response.

The results also show that capillary adhesion is the main source of stiction and damping at the micro-scale. This is an observed and understood phenomenon in small scale devices, where provisions are usually made to mitigate the ingression of moisture into the contacts. In the micro-scale contacts of macro-scale systems such as bearings there is a need for lubrication, not least because this reduces the chance of adhesion of contiguous contacting surfaces through reduction of the surface energy in the scale of asperities. In this diminutive scale the surface energy effects are small compared with the bulk lubricant film action. Any adverse localised effect, such as friction is usually countered by the inclusion of boundary active additive species such as long chain molecules which overlay asperity peaks or adsorb to the surfaces to form low shear strength ultra-thin films [22]. In this manner the effect of adhesion is reduced. The important point is to modify the adhesion map in order to better address the adhesion of asperities in higher loaded lubricated contacts. This constitutes the future direction of the reported research. 


\section{Acknowledgements}

The authors would like to acknowledge the financial support of the Engineering and Physical Sciences Research Council (EPSRC) extended to the Encyclopaedic Program Grant under which some of the research reported here was undertaken.

\section{References}

[1]- Dowson, D., "Developments in lubrication - the thinning film", J. Phys., D: Appl. Phys., 25, 1992, pp. 334-339

[2]- Teodorescu, M. and Rahnejat, H., "Newtonian mechanics in the scale of minutia", Proc. IMechE, Part K: J. Multi-body Dyn., 222(4), 2008, pp. 393-405

[3]- Feynman, R.P., "Plenty of room at the bottom", Transcript of Talk to the American physical Society, Pasadena, USA, December 1959

[4]-Teodorescu, M., Theodossiades, S. and Rahnejat, H., "Impact dynamics of rough and surface protected MEMS gears" Tribology international, 42(2), 2009,pp. 197-205.

[5]- Ashurst, W.R., de Boer, M.P., Carraro, C. and Maboudian, R., "An investigation of sidewall adhesion in MEMS", Appl. Surf. Sci., 212-213, 2003, pp. 735-741.

[6]- Riedo, E., Le'vy, F. and Brune, H., "Kinetics of capillary condensation in nanoscopic sliding friction”, Phys. Rev. Lett., 88(18),2002, 185505.

[7]- de Boer, M.P. and de Boer, P.C.T., "Thermodynamics of capillary adhesion between rough surfaces", J. Colloid Interface Sci., 311, 2007, pp. 171-185.

[8]- Teodorescu, M. and Rahnejat, H., "Dry and wet nano-scale impact dynamics of rough surfaces with or without a self-assembled monolayer", Proc. IMechE, Part N: J. Nanoeng. \& Nanosys., 221 (2), 2007, pp. 49-58.

[9]- Israelachvili, J. N., "Intermolecular and Surface Forces”, Academic Press, New York, 1992

[10]- Al-Samieh, M. and Rahnejat, H., "Ultra-thin lubricating films under transient conditions", J. Phys., D: Appl. Phys. 34(17), 2001: 2610.

[11]- Al-Samieh, M. F. and Rahnejat, H., "Physics of lubricated impact of a sphere on a plate in a narrow continuum to gaps of molecular dimensions", J. Phys. D: Appl. Phys., 35(18), 2002: 2311.

[12]- Gohar, R. and Rahnejat, H., "Fundamentals of Tribology", Imperial College Press, London, 2008. 
[13]- Dowson, D. and Higginson, G.R., “ Elasto-hydrodynamic lubrication”, Pergamon Press, 1977

[14]-Bradley, R.S., "LXXIX: The cohesive force between solid surfaces and the surface energy of solids" The London, Edinburgh, and Dublin Philosophical Magazine \& J. Science, 13(86), 1932, pp. 853-862.

[15]-Johnson, K. L., Kendall, K. and Roberts, A. D., "Surface energy and the contact of elastic solids", Proc. Roy. Soc., Ser. A.: Mathematical and physical sciences, 324.1558, 1971, pp. 301-313.

[16]- Derjaguin, B. V., Muller, V. M. and Toporov, Yu P., "Effect of contact deformations on the adhesion of particles", J. Colloid and interface Sci., 53(2), 1975, pp. 314-326.

[17]- Tabor, D. "Surface forces and surface interactions", J. Colloid and Interface Sci., 58(1), 1977, pp. 2-13.

[18]- Muller, V. M., Yushchenko, V. S. and Derjaguin, B. V., "On the influence of molecular forces on the deformation of an elastic sphere and its sticking to a rigid plane", J. Colloid and Interface Sci., 77(1), 1980, pp. 91-101.

[19]- Greenwood, J. A. "Adhesion of elastic spheres", Proc. Roy. Soc.. Ser. A: Mathematical, Physical and Engineering Sciences, 453.1961, 1997, pp. 1277-1297.

[20]- Maugis, D., "Adhesion of spheres: the JKR-DMT transition using a Dugdale model", J. Colloid and Interface Sci., 150(1), 1992, pp. 243-269.

[21]- Kogut, L. and Etsion, I., "Adhesion in elastic-plastic microcontact", J. Colloid and Interface Sci., 261, 2003,pp. 372-378.

[22]- Chong, W. W. F., Teodorescu, M. and Rahnejat, H., "Nanoscale elastoplastic adhesion of wet asperities", Proc. IMechE, Part J: J. Eng. Tribology, 227(9), 2013, pp. 996-1010

[23]- Greenwood, J. A. and Williamson, J. B. P., "Contact of nominally flat surfaces", Proc. Roy. Soc., Ser. A. Mathematical and Physical Sciences, 295.1442, 1966, pp. 300-319.

[24]- Johnson, K. L., "Mechanics of adhesion", Tribology International, 31(8), 1998, pp. 413418.

[25]- Binquan, L. and Robbins, M.O., "Contact of single asperities with varying adhesion: comparing continuum mechanics to atomistic simulations", Phys. Rev. E, 74(2), 2006, 026111.

[26]- Greenwood, J. A., and Tripp, J. H., "The contact of two nominally flat rough surfaces", Proc. IMechE, J. Mech. Eng. Sci.,185(1 ), 1970-1971, pp. 625-633. 
[27]- Grierson, D. S., Flater, E. E. and Carpick, R.W., "Accounting for the JKR-DMT transition in adhesion and friction measurements with atomic force microscopy" J. Adhesion Sci. and tech., 19(3-5), 2005,pp. 291-311

[28]- Hariri, A., Zu, J. and Ben Mrad, R., "Modeling of wet stiction in microelectromechanical systems (MEMS)", J. Microelectromechanical Systems, 16(5), 2007, pp. 1276-1285.

[29]- Adamson, A. W. and Petry Gast, A., "Physical chemistry of surfaces", Inderscience, 1967, pp. 400-408.

[30] M. Ciavarella, V. Delfine, G. Demelio, A "revitalized" Greenwood and Williamson model of elastic contact between fractal surfaces, J. Mech. Phys. Solids, 54, 2006, pp. 2569-2591.

[31] Afferrante L., Carbone G., Demelio G. Interacting and coalescing Hertzian asperities: A new multiasperity contact model. Wear, 28-33, 2012, pp. 278-279.

[32]- Rahnejat, H., "Tribology and dynamics of engine and powertrain", Woodhead Publishing, Cambridge, 2010.

[33]- Howell-Smith, S., Rahnejat, H., King, P. D. and Dowson, D., "Reducing in-cylinder parasitic losses through surface modification and coating", Proc. IMechE, Part D: J. Automobile Engineering, 228(4), 2014, pp. 391-402

[34]- Etsion, I., "Surface texturing for in-cylinder friction reduction", Woodhead Publishing Ltd, New Delhi (India): 2010. 